\title{
Optimal Numerical Integration on a Sphere
}

\author{
By A. D. McLaren
}

1. Introduction and Summary. This paper discusses the approximation of integrals over the surface of a sphere by formulas of the following form.

$$
\sum_{i=1}^{N} a_{i} f\left(\theta_{i}, \phi_{i}\right) \sim \int f(\theta, \phi) d S .
$$

Little has been published on this subject or on its extension to the solid sphere. The literature is surveyed briefly in Section 7. Most of our space is devoted to formulas invariant with respect to a finite group of rotations of the sphere. We study such formulas by means of the group characters, as does Sobolev [12, 13].

The criterion by which integration formulas are usually judged is that of efficiency. It is defined like this. Consider a system of functions over the domain of integration such as polynomials in Euclidean space or surface harmonics on the sphere. They have properties of completeness and they are ordered in a natural way. Suppose that the integration formula is exact for the first $L$ independent functions and therefore for all linear combinations of them. The efficiency $E$ is the ratio of $L$ to the number of arbitrary constants in the formula. The latter is a fixed multiple (one more than the dimensionality of the domain of integration) of the number $N$ of points at which the integrand is evaluated.

A linear combination of surface harmonics (of degree not more than $p$ ) will be called a spherical polynomial (of degree $p$ ). If we choose to embed the surface of the sphere in Euclidean space of three dimensions, we find that the trace left on the surface by an ordinary polynomial in $x, y$ and $z$ is a spherical polynomial of the same degree. For the surface of the sphere a $p$ th degree integration formula (exact for spherical polynomials of degree $p$ ) has

$$
L=\sum_{m=0}^{p}(2 m+1)=(p+1)^{2}
$$

and

$$
E=\frac{(p+1)^{2}}{3 N}
$$

Efficiency is a useful yardstick and the main part of this paper is written with reference to it. It is not beyond criticism as we shall see later. One suspects that efficiency is used for higher-dimensional regions, largely because it is the natural way of expressing the classical results of mechanical quadrature for the line segment. It is these results that we first attempt to generalize to the surface of the sphere. Generalization from the line segment to the circumference of the unit circle is achieved by replacing the classical arguments [14, Theorem 3.4.1] by their analogues for a complex variable and using the theory of polynomials orthogonal on the unit circle [14, chapter XI]. The classical arguments do not extend to the sphere but we may continue as follows.

Received January 28, 1963. 
In the case of unit weight function the points of the integration formula for the circumference are evenly spaced. In other words, the set of points is invariant under a finite group of rotations of the unit circle. In the case of the sphere we are considering only unit weight function so that it is natural to study sets of points invariant under one of the finite groups of rotations associated with the regular solids. Sections $2-6$ are concerned with this.

Let $g$ be the order of the group. Then we shall see that, apart from any intrinsic merit, the use of an invariant formula reduces by a factor of approximately $g$ the number of independent surface harmonics for which the formula must be made exact. This enables us to find some efficient formulas. They are listed in Table 2. The most spectacular is accurate to the 14th degree, that is exact for 225 independent functions, and uses only 72 points so that $E>1$.

In the case of the circle it is possible to determine an infinite sequence of formulas of increasing accuracy and with $E$ near unity. As far as the writer is aware, no one has shown that this can be done for the sphere or any other two-dimensional region; although $E=\frac{2}{3}$ has been obtained for the sphere by cartesian product methods (see Section 7 ). There is no evidence yet that $E=1$ is a fruitful target in more than one dimension unless we are content with limited accuracy. Nor is $E=1$ a strict upper bound to what may be achieved, as shown by the 14th degree formula cited above. It may be better to seek to generalize the classical results of mechanical quadrature without reference to the efficiency $E$.

In a sequel to this paper it will be shown how restatement in probabilistic terms leads to a concrete problem of minimization with respect to disposition of the sample points (cf. Section 7.1). This method applies also to the circle and yields the classical result.

If approximate integration is to be programmed the number of sample points may be unimportant. To meet this case a sequential procedure based solely on symmetry is outlined in Section 7.2.

The subject of this paper is essentially the wide dispersal of points on the surface of a sphere. It is relevant to interpolation and to certain problems of mathematical statistics, as well as to numerical integration.

2. Existence of Formulas. Properties of group representations assumed here are given by Heine [4, Appendix C.].

The three finite groups $Q_{4}, \delta_{4}, Q_{5}$ of rotations of the sphere are associated respectively with the regular tetrahedron, octahedron-cube and icosahedron-dodecahedron. Let $G$ be a realization of one of these groups, of order $g$, and let $\omega(R)$ be the set of $n$ positions on the sphere that an arbitrary point $R$ takes up under the different rotations of $G$. In general $n=g$, but if $R$ coincides with a vertex of the regular solid, the centroid of a face (vertex of the dual solid) or the mid-point of an edge, then $n<g$.

Now there is induced on the set $\omega(R)$ an $n$-dimensional permutation representation $\{D\}$ of $G$. This may be split into its component irreducible unitary representations $\left\{D_{\lambda}\right\}$; that is, the carrier space $\Omega$ of dimension $n$, is a direct sum

$$
\Omega=\oplus \Omega_{\lambda} ; \quad \lambda=1, \cdots, r
$$

of subspaces $\Omega_{\lambda}$, each invariant under $G$. The dimension of $\Omega_{\lambda}$ is that of $\left\{D_{\lambda}\right\}$ multi- 
plied by the number of times the latter appears in $\{D\}$, which may be zero. The $\Omega_{\lambda}$ are mutually orthogonal, because permutations are unitary transformations. Now $e$, the vector with equal components, is invariant under all permutations and so belongs to $\Omega_{1}$, the subspace subject to the identical representation $\left\{D_{1}\right\}$.

We turn next, for reasons given in the Introduction, to the surface harmonics of fixed degree $m$. These form a $(2 m+1)$-dimensional function-space $V$ invariant under all rotations of the sphere, and therefore under those of $G$. So a $(2 m+1)$ dimensional representation $\{\Delta\}_{m}$ of $G$ is obtained, and $V$ is a direct sum

$$
V=\oplus V_{\lambda} ;
$$

$$
\lambda=1, \cdots, r
$$

where the subspaces $V_{\lambda}$ comprise functions which transform under $G$ according to the inequivalent representations $\left\{D_{\lambda}\right\}$. Consider now the natural projection of $V$ into $\Omega$

$$
V \rightarrow \Omega
$$

whereby every function of $V$ is identified with the $n$-vector of its values at the points of $\omega(R)$. Because $G$ operates both on $V$ and on $\Omega$ then further

$$
V_{\lambda} \rightarrow \Omega_{\lambda} ; \quad \lambda=1, \cdots, r .
$$

If

$$
\begin{aligned}
M \equiv \oplus V_{\lambda} ; & \lambda \neq 1, \quad \operatorname{dim} V_{\lambda} \neq 0, \quad \operatorname{dim} \Omega_{\lambda} \neq 0 \\
N \equiv \oplus V_{\lambda} ; & \lambda \neq 1, \quad \operatorname{dim} V_{\lambda} \neq 0, \quad \operatorname{dim} \Omega_{\lambda}=0
\end{aligned}
$$

then

$$
V=V_{1} \oplus M \oplus N
$$

and the functions of $N$ vanish at every point of $\omega(R)$; while the functions of $M$ are orthogonal, over $\omega(R)$, to $\Omega_{1}$ and thus orthogonal to $e$, that is their average over $\Omega(R)$ is zero. The functions of $V_{1}$ are constant over $\omega(R)$.

The point of this decomposition is that the true value of the integral of any surface harmonic of degree $m$ is zero, except when $m=0$. Now $V_{1}$ does not depend on the particular invariant set $\omega(R)$ under consideration so that an integration formula which assigns equal weights to points in the same invariant set is accurate for the whole of $V$ if it is accurate for $V_{1}$. This statement is trivial when $m=0$ for then $V=V_{1}$. From now on we shall distinguish different values of $m$ by writing $V^{(m)}$ for $V$ and $V_{1}^{(m)}$ for $V_{1}$.

The dimension of $V_{1}^{(m)}$ is the number of times the identical representation appears in $\{\Delta\}_{m}$. Let this be $d_{1}{ }^{(m)}$, for $m=0,1,2, \cdots$. Then an integration formula accurate to the $p$ th degree may be found using just

$$
C_{p} \equiv \sum_{m=0}^{p} d_{1}^{(m)}
$$

invariant sets. In fact almost any $C_{p}$ sets will do, by the following

Theorem. The set of points $R_{1}, R_{2}, \cdots, R_{h}$ on the sphere is said to be a p-adequate $h$-tuple if there exists a pth degree integration formula, invariant under $G$, which uses only $\omega\left(R_{j}\right) ; j=1, \cdots, h$. Then the set of $C_{p}$-tuples which are not $p$-adequate has measure zero (with respect to the natural $C_{p}$-fold product measure). 
Proof. Let a basis of linearly independent functions of

$$
U_{p} \equiv \oplus V_{1}^{(m)} ; \quad m=0,1, \cdots, p
$$

be $f_{i}(\theta, \phi) ; i=1, \cdots, C_{p}$. Then the $C_{p}$-tuple $\left(R_{j}\right) ; j=1, \cdots, C_{p}$ is certainly $p$-adequate if the $C_{p} \times C_{p}$ matrix whose $(i, j)$ th element is the sum of the values of $f_{i}(\theta, \phi)$ at the points of $\omega\left(R_{j}\right)$ is non-singular. Now $V_{1}^{(m)}$ contains only functions that are constant over any $\omega(R)$, so that the determinant that must not vanish is simply

$$
\operatorname{det} A \equiv\left\|f_{i}\left(R_{j}\right)\right\|
$$

The proof is by induction: we assume that $R_{1}, \cdots, R_{h}$ have been chosen, where $1 \leqq h<C_{p}$, so that the first $h$ columns of $A$ are linearly independent and show that almost any choice of $R_{h+1}$ will do. Let $\beta_{i}\left(i=1, \cdots, C_{p}\right)$ be constants, not all zero, such that

$$
\sum_{i=1}^{C_{p}} \beta_{i} f_{i}\left(R_{j}\right)=0 ; \quad j=1, \cdots, h .
$$

If $R$ is any point on the sphere such that the column $f .(R)$ is linearly dependent on the first $h$ columns $f .\left(R_{j}\right), j=1, \cdots, h$, then

$$
\sum_{i=1}^{c_{p}} \beta_{i} f_{i}(R)=0
$$

Now this finite sum of surface harmonics is not identically zero because the $f_{i}(\cdot)$ are the linearly independent functions of $U_{p}$. Hence the set on which it vanishes has measure zero. Thus for almost any $R_{h+1}$ the first $(h+1)$ columns of $A$ are linearly independent. It follows by induction that det. $A$ is non-zero p.p.

3. Calculation of $C_{p}$. Before this theorem can be put to work the numbers $C_{p}$ must be calculated.

The decomposition of $\{\Delta\}_{m}$ is a straightforward matter involving the characters of the $\left\{D_{\lambda}\right\}$ and of $\{\Delta\}_{m}$ itself: see for example Heine [4, page 119]. Consider a rotation of the sphere through an angle $\psi$ about any axis: with this as axis of coordinates the tesseral harmonics

$$
e^{i s \phi} P_{m}{ }^{s}(\cos \theta) ; \quad s=0, \pm 1, \cdots, \pm m
$$

are eigenfunctions of the rotation, with eigenvalues $e^{i s \psi}$. So the character of $\{\Delta\}_{m}$ is

$$
\sum_{s=-m}^{m} e^{i s \psi}=\frac{\sin \left(m+\frac{1}{2}\right) \psi}{\sin \frac{1}{2} \psi} .
$$

Hence

$$
d_{1}^{(m)}=\frac{1}{g} \sum_{k=1}^{g} \frac{\sin \left(m+\frac{1}{2}\right) \psi_{k}}{\sin \frac{1}{2} \psi_{k}}
$$

where the $k$ th group element is a rotation through an angle $\psi_{k}$. The results are 
quite simple because

$$
\delta^{(m)} \equiv d_{1}^{(m)}-\frac{1}{g}(2 m+1)
$$

is periodic in $m$, with period $\frac{1}{2} g$.

An ingenious alternative for (1) is obtained by Sobolev [12]: let $q_{1}, q_{2}, q_{3}$ be the orders of the subgroups of $G$ which give rise respectively to the vertices, faces and edges of the regular solid. Then he finds, for $0 \leqq m \leqq \frac{1}{2} g-1$,

$$
\left.\begin{array}{rlrl}
d_{1}^{(m)} & =0, & & 2 m+1 \leqq g \sum 1 / q \\
& =1, & & 2 m+1>g \sum 1 / q
\end{array}\right\}
$$

where summation extends only to those $q_{i}$ which are not factors of $m$. Extension to larger values of $m$ follows of course from the periodicity of $\delta^{(m)}$.

TABLE 1

\begin{tabular}{|c|c|c|c|c|c|c|c|c|c|c|c|}
\hline & \multicolumn{3}{|c|}{$a_{4}$} & \multicolumn{4}{|c|}{$\mathcal{S}_{4}$} & \multicolumn{4}{|c|}{$a_{5}$} \\
\hline $\begin{array}{l}\text { No. of } \\
\text { elements } \\
\psi_{k}\end{array}$ & \begin{tabular}{l|l}
1 & 3 \\
0 & $\pi$
\end{tabular} & $\begin{array}{l}4 \\
2 \pi / 3\end{array}$ & $\begin{array}{c}4 \\
-2 \pi / 3\end{array}$ & \begin{tabular}{c|c}
1 & 6 \\
0 & $\pi / 2$
\end{tabular} & $\begin{array}{c}8 \\
\pm 2 \pi / 3\end{array}$ & $\begin{array}{l}3 \\
\pi\end{array}$ & $\begin{array}{l}6 \\
\pi\end{array}$ & $\begin{array}{ll}1 & 15 \\
0 & \\
0\end{array}$ & $\begin{array}{l}20 \\
2 \pi / 3\end{array}$ & $\begin{array}{l}12 \\
2 \pi / 5\end{array}$ & $\begin{array}{l}12 \\
4 \pi / 5\end{array}$ \\
\hline
\end{tabular}

Data on Groups

\begin{tabular}{l|l|l|l|l}
\hline & $q_{1}$ & $q_{2}$ & $q_{3}$ & $g$ \\
\hline$a_{4}$ & 3 & 3 & 2 & 12 \\
$S_{4}$ & 4 & 3 & 2 & 24 \\
$a_{5}$ & 5 & 3 & 2 & 60 \\
\hline
\end{tabular}

Tetrahedral Group $a_{4}$

$$
\begin{aligned}
& m: \quad \begin{array}{llllll}
0 & 1 & 2 & 3 & 4 & 5
\end{array}
\end{aligned}
$$

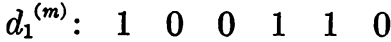

$$
\begin{aligned}
& d_{1}^{(m+6)}=d_{1}^{(m)}+1 \text {. }
\end{aligned}
$$

Octahedral Group $\varsigma_{4}$

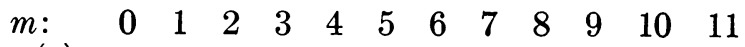

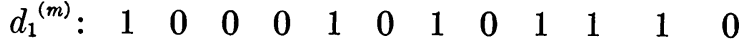

$$
\begin{aligned}
& d_{1}^{(m+12)}=d_{1}^{(m)}+1 \\
& \text { Icosahedral Group } \mathfrak{Q}_{5}
\end{aligned}
$$

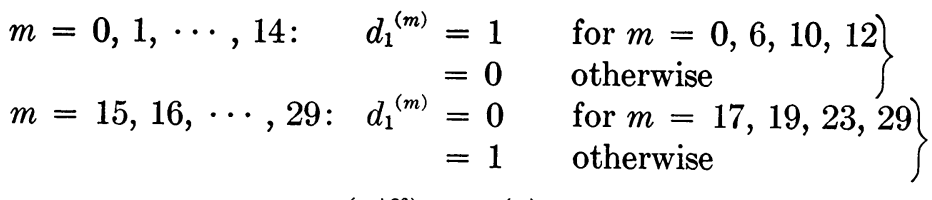

$$
\begin{aligned}
& d_{1}^{(m+30)}=d_{1}^{(m)}+1
\end{aligned}
$$


The $\psi_{k}, q_{i}$ and $d_{1}^{(m)}$ for all three groups appear in Table 1 . The $C_{p}$ may be found simply by counting the $d_{1}^{(m)}$. However even this exertion may be avoided by the following observation.

Let

$$
C_{p}=\frac{1}{g}(p+1)^{2}+\epsilon(p) ; \quad p=0,1,2, \cdots .
$$

Then $\epsilon(p)=0$ when $(p+1)$ is a multiple of $\frac{1}{2} g$ and $\frac{1}{5}<\epsilon(p)<\frac{6}{5}$ otherwise.

This statement, once proved, determines the integer $C_{p}$ exactly. Direct calculation, using Table 1 , shows that the statement is true when $0 \leqq p \leqq \frac{1}{2} g-1$. We show that it is valid without restriction on $p$ by proving that $\epsilon(p)$ is periodic, with period $\frac{1}{2} g$.

$$
\epsilon(p) \equiv C_{p}-\frac{1}{g}(p+1)^{2}=\sum_{m=0}^{p} \delta^{(m)} .
$$

It is convenient to define $C_{-1}=0$, so that $\epsilon(-1)=0$ and

$$
\epsilon\left(p+\frac{1}{2} g\right)-\epsilon(p)=\sum_{m=p+1}^{p+1 / 2 g} \delta^{(m)} \quad(p=-1,0,1,2, \cdots) .
$$

Now this is independent of $p$, because $\delta^{(m)}$ has period $\frac{1}{2} g$, so

$$
\begin{aligned}
\epsilon\left(p+\frac{1}{2} g\right)-\epsilon(p) & =\sum_{m=0}^{1 / 2 g-1} \delta^{(m)} \\
& =\sum_{m=0}^{1 / 2 g-1} d_{1}^{(m)}-\frac{1}{4} g \\
& =0
\end{aligned}
$$

by inspection of Table 1 . Hence $\epsilon(p)$ is periodic, with period $\frac{1}{2} g$.

4. Choice of Invariant Sets. In Figure 1 the spherical triangle $X X^{\prime} X^{\prime \prime}$ matches a face of the tetrahedron, octahedron or icosahedron. $Y$ is the centroid and $Z$ is the mid-point of $X X^{\prime}$. The invariant sets $\omega(R)$ are in one-one correspondence with the points of the closure of triangle $X Y Z$ together with the interior of triangle $X^{\prime} Y Z$. Each has $g$ points, if we exclude the three special sets $\omega(X), \omega(Y)$ and $\omega(Z)$ which together have only $(g+2)$ points. So by $(2)$ an arbitrary $C_{p}$-tuple provides an invariant integration formula with $(p+1)^{2}+g \epsilon(p)$ points. It is $p$-adequate (almost certainly), i.e. it is accurate for

$$
\sum_{m=0}^{p}(2 m+1)=(p+1)^{2}
$$

linearly independent functions.

Now compare what happens if the integration formula is based on $(p+1)^{2}$ arbitrary points, quite unrelated to any rotation group. Take $G_{0}$ to be the (uninteresting) group with just one element. Section 1 still applies and we have in this trivial case $d_{1}{ }^{(m)}=(2 m+1)$ and $C_{p}=(p+1)^{2}$. So the $(p+1)^{2}$ arbitrary points form a $C_{p}$-tuple of "invariant sets" for $G_{0}$ and the Theorem shows that it is almost certainly $p$-adequate. Hence the use of formulas invariant under (non-trivial) rotation groups $G$ does not, by itself, achieve anything. 


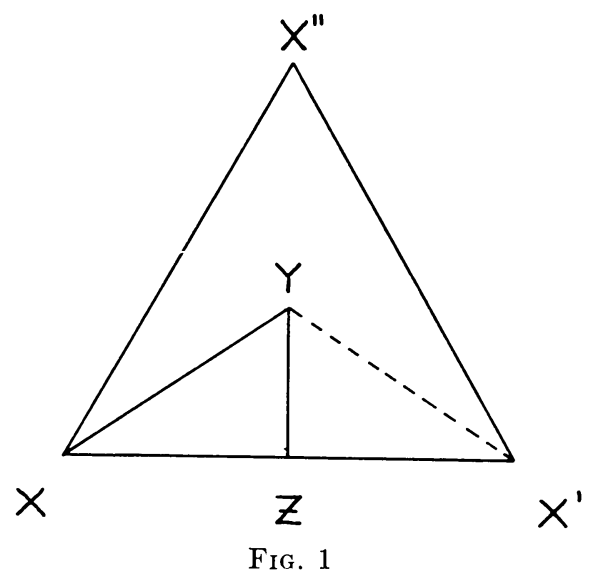

For the octahedron and icosahedron, invariant sets corresponding to points on the boundary of triangle $X Y Z$ are self-antipodal and therefore eliminate odd harmonics automatically, but full use of this property involves restriction to a set of measure zero. Sobolev [13] augments $G$ by reflection in the origin to obtain a group $G^{*}$ of order $2 g$, and considers only sets $\omega^{*}(R)$ invariant with respect to $G^{*}$. These are in one-one correspondence with the points of the closure of triangle $X Y Z$. Unless $R$ is $X, Y$ or $Z, \omega^{*}(R)$ has $g$ or $2 g$ points according as $R$ is or is not on the boundary of triangle $X Y Z$. Sobolev considers two series of formulas, of increasing adequacy, and calculates their efficiency. This depends on the proportion (which tends to zero) of points lying on the boundary of triangle $X Y Z$. So even these are no more efficient, asymptotically, than if the points were chosen at random.

Our theory seems to be useful only where it simplifies the proper choice of invariant sets to obtain efficient formulas. We saw at the beginning of this section that the efficiency of an integration formula based on $C_{p}$ arbitrary invariant sets could not be expected to exceed

$$
\frac{(p+1)^{2}}{3 g C_{p}}=\frac{(p+1)^{2}}{3\left[(p+1)^{2}+g \epsilon(p)\right]} \leqq \frac{1}{3} .
$$

Consider the choice of a formula based on $h$ general invariant sets $\omega\left(R_{i}\right)$ and $h^{\prime}$ special ones, where $h$ and $h^{\prime}$ are fixed integers and $0 \leqq h^{\prime} \leqq 3$. The $R_{i}$ vary within a two-dimensional region and so each general set may be said to have two positional degrees of freedom. The weights to be assigned to each set are also (as before) at our disposal so that the formula has altogether

$$
2 h+\left(h+h^{\prime}\right)=3 h+h^{\prime}
$$

degrees of freedom. We hope that each degree of freedom can be used to bring one more independent function within the formula's domain of accuracy. We adopt the Working Hypothesis: An invariant integration formula accurate for spherical polynomials of degree not more than $p$ may be found using just $h$ general invariant sets and $h^{\prime}$ special sets, if

$$
3 h+h^{\prime}=C_{p}
$$


To try and construct the formula seems to be the simplest way of finding out whether, for a particular value of $p$, the hypothesis is true. Assuming its validity for the moment we shall consider the efficiency

$$
E \equiv \frac{(p+1)^{2}}{3 N}
$$

of conceivable formulas satisfying (3), where $N$ is the total number of points used. $E \rightarrow 1$ as $p \rightarrow \infty$, for

$$
N=g h+O(1)
$$

and so

$$
\begin{aligned}
3 N & =3 g h+O(1)=g\left(C_{p}-h^{\prime}\right)+O(1) \\
& =g C_{p}+O(1) \\
& =(p+1)^{2}+O(1) .
\end{aligned}
$$

We saw just now that without the positional degrees of freedom $3 N=3 g C_{p}=$ $3(p+1)^{2}+O(1)$ so that $E \rightarrow \frac{1}{3}$.

In fact $E>1$ for some finite values of $p$, as we see below.

1) $h^{\prime}=0: 3 h=C_{p}$ and $N=g h=\frac{1}{3} g C_{p}$, so $3 N=(p+1)^{2}+g \epsilon(p) \geqq(p+1)^{2}$ with equality (i.e. $E=1$ ) if and only if $\epsilon(p)=0$, that is when $(p+1)$ is a multiple of $\frac{1}{2} g$.

2) $h^{\prime}=1: 3 h+1=C_{p}$ and $N=g h+g / q_{1}$ because the smallest special set will be used, of course. So

$$
\begin{aligned}
3 N & =g\left(C_{p}-1\right)+3 g / q_{1} \\
& =(p+1)^{2}+g\left(\epsilon(p)-\left(1-3 / q_{1}\right)\right)
\end{aligned}
$$

3) $h^{\prime}=2$ : Similar argument gives

$$
\begin{aligned}
3 N & =(p+1)^{2}+g\left(\epsilon(p)-\left(2-3 / q_{1}-3 / q_{2}\right)\right) \\
& =(p+1)^{2}+g\left(\epsilon(p)-\left(1-3 / q_{1}\right)\right)
\end{aligned}
$$

because $q_{2}=3$ for all three groups.

4) $h^{\prime}=3: 3 h+3=C_{p}$. The three special sets have together $(g+2)$ points and would be better replaced by another general set. So $E$ is strictly less than in case 1 ), that is $E<1$.

When $\epsilon(p)=0, C_{p}=\frac{1}{g}(p+1)^{2}$. Since $g$ is divisible by 3 and $C_{p}$ is an integer $(p+1)^{2}$ is divisible by 3 . So $(p+1)^{2}$ is divisible by $3^{2}$. But $g$ is not divisible by $3^{2}$. Hence $C_{p}$ is a multiple of 3 .

So from case 1$)$ every value of $p$ such that $(p+1)$ is a multiple of $\frac{1}{2} g$ gives a formula with $E=1$. In cases 2 ) and 3) $E \geqq 1$ if

$$
\epsilon(p) \leqq 1-3 / q_{1}=0, \frac{1}{4}, \frac{2}{5}
$$

for $Q_{4}, S_{4}$ and $Q_{5}$ respectively. Now for $S_{4} \epsilon(p) \leqq \frac{1}{4}$ only when $\epsilon(p)=0$. So cases 2) and 3) cannot give $E \geqq 1$, for $a_{4}$ or $\delta_{4}$, because $\epsilon(p)=0$ implies that $C_{p}$ is a multiple of 3 . For $a_{5}, \epsilon(p)=\frac{2}{5}$ when $p+1 \equiv \pm 6(\bmod 30)$ and $0<\epsilon(p)<\frac{2}{5}$ when $p+1 \equiv \pm 10,15(\bmod 30)$. These give formulas with $E \geqq 1$ only when 
$C_{p}$ is not a multiple of 3 . By the periodicity of $\epsilon(p)$

$$
\begin{aligned}
C_{p+30}-C_{p} & =\frac{1}{60}(p+1+30)^{2}-\frac{1}{60}(p+1)^{2} \\
& \equiv p+1(\bmod 3) .
\end{aligned}
$$

A short calculation shows that cases 2) and 3) give $E \geqq 1$ for $Q_{5}$ when

$$
p+1 \equiv \pm 6,15(\bmod 30)
$$

or

$$
p+1 \equiv \pm 10, \pm 20(\bmod 90) \text {. }
$$

Combining these results with those of case 1 ) we list finally those values of $p$ for which the Working Hypothesis (if true) predicts an invariant integration formula of degree $p$ with $E \geqq 1$.

$$
\begin{array}{ll}
p+1 \equiv 0(\bmod 6) & E=1 \\
p+1 \equiv 15(\bmod 30) & E>1 \\
p+1 \equiv \pm 10(\bmod 90) & \\
p+1 \equiv \pm 20(\bmod 90) . &
\end{array}
$$

In the next section necessary and sufficient conditions are given for a formula to be accurate up to degree $p$. In section 6 these are used to construct some lowdegree formulas. It will then be seen that the Working Hypothesis is frequently valid and that $E>1$ does occur.

5. Conditions for a $p$ th-Degree Formula. Let $A_{1}, \cdots, A_{t}$ be arbitrary points on the unit sphere and let $\gamma_{r s}$ be the arc-length $A_{r} A_{s}$. Let $a_{1}, \cdots, a_{t}$ be real weights. Then $\sum_{r=1}^{t} a_{r} f\left(A_{r}\right)$ vanishes for all surface harmonics $f(\cdot)$ of degree $m$ if and only if

$$
\sum_{r=1}^{t} \sum_{s=1}^{t} a_{r} a_{s} P_{m}\left(\cos \gamma_{r s}\right)=0
$$

This follows from the addition theorem for spherical harmonics

$$
\frac{2 m+1}{4 \pi} P_{m}\left(\cos \gamma_{r s}\right)=\sum_{n=-m}^{m} T_{m}^{n}\left(A_{r}\right)\left(T_{m}{ }^{n}\left(A_{s}\right)\right)^{*}
$$

where the $T_{m}{ }^{n}(\cdot)$ are normalized tesseral harmonics.

Now let $R_{1}, \cdots, R_{k}$ be any $k$-tuple; and define for $i, j=1, \cdots, k$ :

$$
\begin{aligned}
\pi_{i j}^{(m)}=\sum_{r=1}^{n_{i}} \sum_{s=1}^{n_{j}} P_{m}\left(\cos A_{r} B_{s}\right) ; & A_{r} \in \omega\left(R_{i}\right) \\
& B_{s} \in \omega\left(R_{j}\right) .
\end{aligned}
$$

$n_{i}=g$ except when $\omega\left(R_{i}\right)$ is one of the special sets. The $k \times k$ matrix

$$
\mathrm{II}^{(m)} \equiv\left[\pi_{i j}^{(m)}\right]
$$

is symmetric and non-negative definite (by the addition theorem) and the $k$ in- 
variant sets, with vector of weights $a$, provide a $p$ th degree formula if and only if

$$
a^{\prime} \Pi^{(m)} a=0 ; \quad m=1,2, \cdots, p
$$

that is

$$
\Pi^{(m)} a=0 ; \quad m=1,2, \cdots, p .
$$

In fact $\sum a_{i} n_{i} \neq 0$ is also necessary, but it is impossible to overlook the failure of this condition in practice, so we shall not mention it again.

LEMMA. The rank of $\Pi^{(m)}$ is at most $d_{1}{ }^{(m)}$.

Proof. A non-zero vector of weights may be found for any $h$-tuple of invariant sets, where $h>d_{1}{ }^{(m)}$, to eliminate the surface harmonics of degree $m$. This follows from Section 1 , since $d_{1}{ }^{(m)}$ homogeneous equations in $\left(d_{1}{ }^{(m)}+1\right)$ unknowns have always a non-zero solution. Hence, by the argument of the present Section, all principal minors of $\Pi^{(m)}$ larger than $d_{1}^{(m)} \times d_{1}{ }^{(m)}$ vanish. But $\Pi^{(m)}$ is symmetric, so that its rank is at most $d_{1}^{(m)}$.

In particular when $d_{1}{ }^{(m)}=1$

$$
\pi_{i j}^{(m)}=\epsilon_{i} \epsilon_{j} \sqrt{ }\left(\pi_{i i}^{(m)} \pi_{j j}^{(m)}\right) ; \quad \quad \epsilon_{i}= \pm 1 .
$$

So if $d_{1}^{(m)} \leqq 1(m=1, \cdots, p)$ the conditions $a^{\prime} \Pi^{(m)} a=0$ for a $p$ th degree formula become

$$
\left.\sum_{i=1}^{k} a_{i} \epsilon_{i} \sqrt{ } \pi_{i i}^{(m)}=0 ; \quad \begin{array}{l}
1 \leqq m \leqq p \\
d_{1}^{(m)}=1
\end{array}\right\}
$$

since values of $m$ for which $d_{1}^{(m)}=0$ impose no constraint. Returning to the definition we find that $\pi_{i i}^{(m)}$ is a complicated function of the position of $R_{i}$. The special set $\omega(X)$ will now play the rôle of a pivot, to simplify the form of the constraints. Define $R_{0} \equiv X$, which may or may not be a member of the $k$-tuple. We have of course

$$
\left.\pi_{i 0}^{(m)}=\epsilon_{i} \epsilon_{0} \sqrt{ }\left(\pi_{i i}^{(m)} \pi_{00}^{(m)}\right) ; \quad \begin{array}{l}
i=1, \cdots, k \\
d_{1}{ }^{(m)}=1
\end{array}\right\}
$$

and so

$$
\left.\sum_{i=1}^{k} a_{i} \pi_{i 0}^{(m)}=0 ; \quad \begin{array}{l}
1 \leqq m \leqq p \\
d_{1}{ }^{(m)}=1
\end{array}\right\}
$$

This is sufficient provided $\pi_{00}^{(m)}$ vanishes only when $d_{1}^{(m)}$ does. It can be shown that, for the tetrahedron $\pi_{00}^{(m)}=0$ implies $d_{1}^{(m)}=0$. We omit a proof because one may be obtained by the methods which are applied below to the other two groups.

For the octahedron and icosahedron, $\pi_{00}^{(m)}$ vanishes for all odd $m$ because $\omega(X)$ is self-antipodal. So if there are odd harmonics to be eliminated they must be treated separately. If $m$ is even and $d_{1}^{(m)} \geqq 1$ then $\pi_{00}^{(m)}$ could vanish only by accident: we shall show that this never happens.

Group $\mathrm{S}_{4}$ : The distance between two vertices of the octahedron is either 0 , $\pi / 2$ or $\pi$, and

$$
\pi_{00}^{(2 r)}=6 \times 2\left(1+2 P_{2 r}(0)\right) ; \quad r=0,1,2, \cdots
$$


The inequality:

$$
(\sin \theta)^{1 / 2} \cdot\left|P_{n}(\cos \theta)\right|<\left(\frac{2}{\pi}\right)^{1 / 2} n^{-1 / 2}
$$

given in Szegö [14, page 163], shows that $\left|P_{n}(0)\right|<\frac{1}{2}$ when $n \geqq 3$. So $\pi_{00}^{(2 r)} \neq 0$, when $r \geqq 2$. But $d_{1}^{(2)}=0$.

Group $Q_{5}$ : The distance between two vertices of the icosahedron is either $0, \alpha, \pi-\alpha$ or $\pi$ and

$$
\pi_{00}^{(2 r)}=12 \times 2\left(1+5 P_{2 r}(\cos \alpha)\right) ; \quad r=0,1,2, \cdots .
$$

Since $d_{1}^{(2)}=0, \pi_{00}^{(2)}=0$; so $\cos ^{2} \alpha=\frac{1}{5}$. The inequality gives $\left|P_{n}(1 / \sqrt{ } \tilde{j})\right|<\frac{1}{5}$ when $n \geqq 18$. Thus $\pi_{00}^{(2 r)} \neq 0$ when $r \geqq 9$. Since $d_{1}^{(2)}=d_{1}{ }^{(4)}=d_{1}{ }^{(8)}=d_{1}{ }^{(14)}=0$, only the cases $2 r=6,10,12,16$ remain.

Now the coefficients of a Legendre polynomial, when multiplied by a suitable power of 2 , are integers. So, for $r \geqq 2,5 P_{2 r}(1 / \sqrt{ } 5)$ can be an integer only if the coefficient of the leading term of $P_{2 r}(\cdot)$ contains a power of 5 . If it contains a single factor of 5 , then the next coefficient must not contain a power of 5 . The leading terms of $P_{n}(\mu)$ are:

$$
\frac{1 \cdot 3 \cdot 5 \cdot \cdots(2 n-1)}{1 \cdot 2 \cdot 3 \cdot \cdots n}\left\{\mu^{n}-\frac{n(n-1)}{2(2 n-1)} \mu^{n-2}+\cdots\right\}
$$

and the first coefficient has no power of 5 for $n=6,10,12$. For $n=16$ there is a single factor 5 , but this is present also in the second coefficient. So $\tilde{j} P_{2 r}(1 / \sqrt{ } \tilde{5})$ is not an integer for $2 r=6,10,12,16$.

Hence $\pi_{00}^{(2 r)} \neq 0$ for $2 r=6,10,12,16$.

6. Construction of Formulas. We can now find some formulas whose efficiency is near unity. The tetrahedral group is not considered because every tetrahedral invariant set can be supplemented by another to form an invariant set of an octahedral realization (since two mutually antipodal tetrahedra make a cube). Nor do we look for formulas with more than one general invariant set $(h>1)$, because the calculations become more complex. The results obtained are listed in Table 2 .

6.1 Special Sets Only $(h=0)$. Obvious at once are formulas based on a single special set $\left(h^{\prime}=1\right)$. The existence of the others must be proved by calculation and a first step is to find the distances from a point of $\omega\left(R_{0}\right) \equiv \omega(X)$ to the points of $\omega(Y)$ and $\omega(Z)$.

Group $\varsigma_{4}$ : The distance between a vertex of the octahedron and one of the cube is $\phi$ or $\pi-\phi$ where, since $d_{1}^{(2)}=0, \pi_{X Y}^{(2)}=0$ (with obvious notation). Thus $P_{2}(\cos \phi)=0$, so:

$$
\cos ^{2} \phi=\frac{1}{3} \text {. }
$$

The distance between a vertex of the octahedron and the mid-point of an edge is $\psi, \pi / 2$ or $\pi-\psi$ where $\pi_{X Z}^{(2)}=0$, that is:

$$
2 P_{2}(\cos \psi)+P_{2}(0)=0
$$


SO

$$
\cos ^{2} \psi=\frac{1}{2}
$$

Group $a_{5}$ : The distance between a vertex of the icosahedron and one of the dodecahedron is $\alpha, \beta, \pi-\beta$ or $\pi-\alpha$ where, since $d_{1}^{(2)}=d_{1}^{(4)}=0, \pi_{X Y}^{(2)}=\pi_{X Y}^{(4)}=0$.

TABLE 2

Summary of Formulas

\section{Tetrahedral Group}

The four vertices of the tetrahedron, that is the special set $\omega(X)$, provide a second-degree formula. The group is not exploited any further for the reason given at the beginning of Section 6 .

\begin{tabular}{|c|c|c|c|c|c|c|c|c|c|}
\hline \multicolumn{10}{|c|}{ Octahedral Group } \\
\hline$p$ & $C_{p}$ & $N$ & $E$ & $a_{X}$ & $a_{Y}$ & $a_{z}$ & $a$ & $R$ & $\begin{array}{l}\text { Pri- } \\
\text { ority }\end{array}$ \\
\hline 3 & 1 & $\begin{array}{r}6 \\
8 \\
12\end{array}$ & 0.89 & 1 & 1 & 1 & & & K S \\
\hline 5 & 2 & $\begin{array}{l}14 \\
18 \\
20\end{array}$ & & $\begin{array}{l}8 \\
1\end{array}$ & $\begin{array}{l}9 \\
9\end{array}$ & $\begin{array}{r}2 \\
-16\end{array}$ & & & $\begin{array}{l}\mathrm{K} \\
\mathrm{K} \\
\mathrm{S}\end{array}$ \\
\hline 7 & 3 & $\begin{array}{l}24 \\
26\end{array}$ & 0.89 & 40 & 27 & 32 & 1 & $(0.866,0.423,0.267)$ & $\mathrm{K}$ \\
\hline 8 & 4 & 30 & 0.90 & 16 & & & 21 & $(0.819,0.517,0.251)$ & \\
\hline 11 & 6 & 50 & 0.96 & 9216 & 15309 & 16384 & 14641 & $(0.906,0.302,0.302)$ & \\
\hline \multicolumn{10}{|c|}{ Icosahedral Group } \\
\hline 5 & 1 & \begin{tabular}{l|}
12 \\
20 \\
30
\end{tabular} & 1.00 & 1 & 1 & 1 & & & $\begin{array}{l}\mathrm{F} \\
\mathrm{F}\end{array}$ \\
\hline 9 & 2 & \begin{tabular}{l|l|}
32 & \\
42 & 50
\end{tabular} & 1.04 & $\begin{array}{l}25 \\
25\end{array}$ & $\begin{array}{l}27 \\
27\end{array}$ & $\begin{array}{r}32 \\
-32\end{array}$ & & & $\begin{array}{l}\mathrm{F} S \\
\mathrm{~S}\end{array}$ \\
\hline 11 & 3 & 62 & & 625 & 243 & 512 & & & \\
\hline 14 & 4 & 72 & 1.04 & 125 & & & 143 & see Section 6.42 & \\
\hline
\end{tabular}

$p$ : degree of spherical polynomial for which formula is exact.

$C_{p}$ : number of degrees of freedom of formula.

$N$ : number of points.

$E$ : efficiency (listed only once for each value of $p$ ).

$a_{X}, a_{Y}, a_{Z}, a$ : weights assigned to points of $\omega(X), \omega(Y), \omega(Z), \omega(R)$ respectively.

$R$ : Cartesian co-ordinates of generator of $\omega(R)$. 
Hence:

$$
P_{2}(\cos \alpha)+P_{2}(\cos \beta)=P_{4}(\cos \alpha)+P_{4}(\cos \beta)=0,
$$

so

$$
\cos ^{2} \alpha, \cos ^{2} \beta=\frac{1}{15}(5 \pm 2 \sqrt{ } 5) .
$$

The distance between a vertex of the icosahedron and the mid-point of an edge is $\gamma, \delta, \pi / 2, \pi-\delta$ or $\pi-\gamma$ where $\pi_{X Z}^{(2)}=\pi_{X Z}^{(4)}=0$, that is:

$$
P_{2}(\cos \gamma)+P_{2}(\cos \delta)+P_{2}(0)=P_{4}(\cos \gamma)+P_{4}(\cos \delta)+P_{4}(0)=0,
$$

so

$$
\cos ^{2} \gamma, \quad \cos ^{2} \delta=\frac{1}{10}(5 \pm \sqrt{ } 5) .
$$

The three designs for each group that use just two special sets may now be found. So may the designs using three special sets. The calculation is given only for the 11th degree icosahedral design.

We demand:

$$
a_{X} \pi_{X X}^{(m)}+a_{Y} \pi_{Y X}^{(m)}+a_{Z} \pi_{Z X}^{(m)}=0 ; \quad m=6,10 .
$$

It is true in any case for $m=2,4,8$. Consider the expansion, when $m$ is even, of $(\cos \theta)^{m}$ in Legendre polynomials $P_{n}(\cos \theta):$ the constant term is $\frac{1}{m+1}$. It follows that we require for $m$ even and $\leqq 10$ :

$a_{X} \sum_{\omega(X)} \sum_{\omega(X)}\left(\cos \theta_{r s}\right)^{m}+a_{Y} \sum_{\omega(X)} \sum_{\omega(Y)}\left(\cos \theta_{r s}\right)^{m}+a_{Z} \sum_{\omega(X)} \sum_{\omega(Z)}\left(\cos \theta_{r s}\right)^{m}$

$$
=\frac{1}{m+1}\left(12^{2} \cdot a_{X}+12.20 a_{Y}+12.30 a_{Z}\right)
$$

where the angles involved have just been found.

Putting $m=6,10$ we obtain

$$
\begin{array}{r}
a_{X} / 5^{2}-a_{Y} / 3^{3}-a_{Z} / 2^{5}=0 \\
7^{2} a_{X} / 5^{4}-17 a_{Y} / 3^{5}-a_{Z} / 2^{4}=0
\end{array}
$$

and so

$$
a_{X}: a_{Y}: a_{Z}=5^{4}: 3^{5}: 2^{9}
$$

6.2 Octahedral Group: $h=1$. The simplest formula is one with a single, general invariant set but no special sets. The conditions are

$$
\pi_{i 0}^{(m)}=0 ; \quad 1 \leqq m \leqq 7
$$

For any $m>0, \pi_{i 0}^{(m)}$ vanishes somewhere on the sphere because, regarded as a function of $R_{i}$, it is a surface harmonic of degree $m>0$ so that its integral over the sphere vanishes. Two may or may not vanish together, but there is certainly no reason to expect a simultaneous zero of more than two. So with $g=24$ points we can hope for a 7 th degree formula.

Let $\pm \mu_{1}, \pm \mu_{2}, \pm \mu_{3}$ be the cosines of the distances from a point of the required 
invariant set to the octahedral points. Let $\nu_{j} \equiv \mu_{j}{ }^{2}(j=1,2,3)$. Then

$$
\pi_{i 0}^{(m)}=0 ; \quad m=2,4,6
$$

implies

$$
\sum_{j=1}^{3} \nu_{j}^{n}=\frac{3}{2 n+1}
$$$$
n=1,2,3
$$

by the expansion of $\mu^{2 n}$ in Legendre polynomials. Hence the $\nu_{j}$ are the roots of

$$
105 \nu^{3}-105 \nu^{2}+21 \nu-1=0
$$

which are, nearly, 0.750, 0.179, 0.071. Since these squares of the direction cosines are real, lie between 0 and 1 , and sum to unity, the set exists. In fact there are two mutually antipodal sets. Either gives a 7 th degree design.

Two more formulas based on the octahedral group are found. The first is 8 th degree with 30 points $\left(h=1 ; h^{\prime}=1\right)$.

$$
a_{0} \pi_{00}^{(m)}+a \pi_{i 0}^{(m)}=0 ; \quad m=2,4,6,8 .
$$

With notation as before and $S_{n} \equiv \sum_{j=1}^{3} \nu_{j}{ }^{n}$, then $S_{4}, S_{3}$ and $S_{2}$ can be expressed as linear functions of the ratio of weights $a / a_{0}$. Since $S_{1}=1$, the cubic whose roots are the $\nu_{j}$ has just two unknown coefficients and $S_{4}, S_{3}, S_{2}$ have an alternative expression in terms of these. When the unwanted solution $R_{i}=X$ is rejected the equations can be solved and the cubic is

$$
441 \nu^{3}-441 \nu^{2}+105 \nu-5=0
$$

with roots $0.670,0.267,0.063$ approximately, and $a_{0}: a=16: 21$. Again there are two equivalent, mutually antipodal formulas.

The case $\left(h=1 ; h^{\prime}=2\right)$ could give a 9th degree formula with (at least) 38 points. We do not investigate this because a 9 th degree formula with fewer points has already been found, based on the icosahedral group.

The case $\left(h=1 ; h^{\prime}=3\right)$ is more valuable so we solve it. The only novelty is that an odd harmonic must be eliminated. This is done by confining $R_{i}$ to the boundary of triangle $X Y Z$ ( see Figure 1, Section 3). The "even" conditions

$$
a_{01} \pi_{00}^{(m)}+a_{1} \pi_{01}^{(m)}+a_{2} \pi_{02}^{(m)}+a \pi_{i 0}^{(m)}=0 ; \quad m=4,6,8,10
$$

give, after elimination of the weights, a linear equation in $S_{5}, S_{4}, S_{3}, S_{2}$. There are now two possibilities: if $R_{i}$ is on $X Z$ it is distance $\pi / 2$ from $X^{\prime \prime}$ and one root of the cubic is zero. This leads only to $R_{i}=X$ or $R_{i}=Z$, both of which are unacceptable. So $R_{i}$ must be tried on $X Y$ or $Y Z$, implying that the roots have the form $\nu, \nu, 1-2 \nu$. The linear equation in the $S_{n}$ is now a quintic in $\nu$ but after rejection of the solutions corresponding to $R_{i}=X, R_{i}=Z$ and $R_{i}=Y$ (twice) only

$$
\nu=1 / 11
$$

remains. The weights $a_{0}: a_{1}: a_{2}: a$ are as $2^{10} \cdot 3^{2}: 3^{7} \cdot 7: 2^{14}: 11^{4}$.

6.3. Icosahedral Group: $h=1$. An 11th degree formula with one general set alone $\left(h^{\prime}=0\right)$ is expected. The conditions are

$$
\pi_{i 0}^{(m)}=0 ; \quad 1 \leqq m \leqq 11 .
$$


Let $\pm \mu_{j}(j=1, \cdots, 6)$ now be the cosines of the distances from a point of the required invariant set to the vertices of the icosahedron. Let $\nu_{j} \equiv \mu_{j}{ }^{2}(j=1, \cdots, 6)$. Then

$$
\pi_{i 0}^{(m)}=0 ; \quad m=2,4,6,8,10
$$

implies

$$
S_{n} \equiv \sum_{j=1}^{6} \nu_{j}^{n}=\frac{6}{2 n+1} ; \quad n=1,2,3,4, \bar{j}
$$

by the expansion of $\mu^{2 n}$ in Legendre polynomials. $S_{6}$ can be found from the remaining condition $\pi_{i 0}^{(14)}=0$ which is satisfied vacuously, since $d_{1}^{(14)}=0$.

The sixth degree polynomial whose roots are the $\nu_{j}$ is now determined, but when it is solved only two of the roots are found to be real. Hence there is no 11th degree formula based on a single invariant set of the icosahedral group.

This instance of failure of the Working Hypothesis of Section 4 is interesting because of its success in all the other cases investigated (those listed in Table 2).

Finally, we solve the case $\left(h=1 ; h^{\prime}=1\right)$ to obtain a 14th degree formula with 72 points. As predicted in Section 3 , it is super-efficient $(E>1)$. The solution is determined by

$$
a_{0} \pi_{00}^{(m)}+a \pi_{i 0}^{(m)}=0 ; \quad m=2,4, \cdots, 14,
$$

and the calculation, although lengthy, is similar to those already described. After rejecting $R_{i}=X$ we find

$$
a_{0}: a=125: 143
$$

and

$2556125 \nu^{6}-5112250 \nu^{5}+3578575 \nu^{4}-1043900 \nu^{3}+115115 \nu^{2}-3562 \nu+9=0$.

This was solved on EDSAC II, with this result:

$$
\begin{array}{lll}
\nu_{1}=0.83186 & \nu_{3}=0.41189 & \nu_{5}=0.044731 \\
\nu_{2}=0.56075 & \nu_{4}=0.14800 & \nu_{6}=0.0027682
\end{array}
$$

We have still to show that these six numbers are the squares of the cosines of the distances from some point $R$ on the sphere to the vertices of the icosahedron. If so then we have a 14 th degree formula based on $\omega(X)$ and $\omega(R)$. Now $\cos ^{-1}\left(\nu_{1}^{1 / 2}\right)$ is just greater than $24^{\circ}$ while $\cos ^{-1}\left(\nu_{2}^{1 / 2}\right)$ is just greater than $41^{\circ}$. Thus

$$
\cos ^{-1}\left(\nu_{1}^{1 / 2}\right)+\cos ^{-1}\left(\nu_{2}^{1 / 2}\right)>65^{\circ} \text {. }
$$

The distance between two neighboring vertices of the icosahedron is $\cos ^{-1}\left(\tilde{J}^{-1 / 2}\right)$ which is just less then $64^{\circ}$ (Section 5). So there is a point $R$ such that $\nu_{1}$ and $\nu_{2}$ are the squares of the cosines of the distances from it to two vertices of the icosahedron. Now the sixth degree polynomial satisfied by the $\nu_{j}(j=1, \cdots, 6)$ was constructed so that the sums $S_{n} \equiv \sum_{j=1}^{6} \nu_{j}{ }^{n}$ satisfied four vacuous conditions, that is conditions satisfied whatever position $R_{i}$ takes on the sphere. It is shown in the next Section that these conditions ensure that the remaining $\nu_{j}(j=3, \cdots, 6)$ are the squares of the cosines of the distances of the point $R$ (determined by $\nu_{1}$ and $\nu_{2}$ ) to the remaining vertices of the icosahedron. 
So we have found a super-efficient formula accurate for spherical polynomials of degree not more than fourteen. In Section 6.42 we show how to find the co-ordinates of the required points with respect to rectangular axes with origin at the center of the sphere. This will provide also a useful numerical check on the calculation. The co-ordinates are listed in Table 2.

The cases $\left(h=1 ; h^{\prime}=2\right)$ and $\left(h=1 ; h^{\prime}=3\right)$ for the icosahedral group have not been investigated.

6.4. Icosahedral Co-ordinates. The icosahedral co-ordinates of a point on the sphere are defined to be the squares of the cosines of the distances from that point to the twelve vertices of the icosahedron. Since these vertices are antipodal in pairs a point has just six icosahedral co-ordinates. They are of course the $\nu_{j}$ of the previous Section.

\subsection{Existence of a point with given icosahedral co-ordinates.}

Remark: Consider a sextic equation with $\operatorname{roots} \nu_{j}^{\prime}(j=1, \cdots, 6)$ which are real and lie in $[0,1]$. Suppose the equation has been constructed as if its roots were the icosahedral co-ordinates of a point on the sphere, that is

$$
\pi_{i 0}^{(m)}=0 ; \quad m=2,4,8,14 .
$$

Suppose further that there is a point on the sphere with icosahedral co-ordinates $\nu_{j}^{\prime \prime}(j=1, \cdots, 6)$ two of which coincide with two of the roots. Say $\nu_{1}^{\prime \prime}=\nu_{1}^{\prime}$ and $\nu_{2}^{\prime \prime}=\nu_{2}^{\prime}$; then the other icosahedral co-ordinates $\nu_{j}^{\prime \prime}(j=3, \cdots, 6)$ coincide with the other roots $\nu_{j}^{\prime}(j=3, \cdots, 6)$.

Outline of Proof: The values $m=2,4,8,14$ are of course those even ones for which $d_{1}^{(m)}=0$. The four conditions $\pi_{i 0}^{(m)}=0$ are linear in the sums

$$
S_{n}{ }^{\prime} \equiv \sum_{j=1}^{6} \nu_{j}^{\prime n} ; \quad n=1,2, \cdots, 7
$$

of the powers of the roots of the sextic equation. The conditions are satisfied also by the same functions

$$
S_{n}^{\prime \prime} \equiv \sum_{j=1}^{6} \nu_{j}^{\prime \prime} ; \quad n=1,2, \cdots, 7
$$

of the $\nu_{j}^{\prime \prime}$, because the $\nu_{j}^{\prime \prime}$ are icosahedral co-ordinates. For the same reason $0 \leqq \nu_{j}^{\prime \prime} \leqq 1 ;$ and $0 \leqq \nu_{j}^{\prime} \leqq 1$ by hypothesis.

We have therefore to show that the solution of the equations

$$
\pi_{i 0}^{(m)}=0 ; \quad m=2,4,8,14
$$

in unknowns $\nu_{j}(j=1, \cdots, 6)$ is unique when $\nu_{1}$ and $\nu_{2}$ are given and $0 \leqq \nu_{j} \leqq 1$ $(j=1, \cdots, 6)$. The quartic whose roots are the $\nu_{j}(j=3, \cdots, 6)$ is constructed as follows:

$$
\pi_{i 0}^{(2)}=\pi_{i 0}^{(4)}=0 \text { determines } \sum_{j=3}^{6} \nu_{j} \text { and } \sum_{j=3}^{6} \nu_{j}^{2}
$$

and thus two of the coefficients. By use of the relations between the elementary symmetric functions and the sums of the powers of the roots the remaining con- 
ditions $\pi_{i 0}^{(8)}=\pi_{i 0}^{(14)}=0$ yield one linear and one quadratic equation in the remaining coefficients $\alpha$ and $\beta$ of the quartic. We find at last that $\alpha$ satisfies a quadratic the sum of whose roots is

$$
\sigma \equiv \frac{1}{5}\left(436-14 f+40 g+20 f^{2}-80 f g-10 f^{3}\right)
$$

where $0 \leqq f \equiv \nu_{1}+\nu_{2}<2$ and $0 \leqq g \equiv \nu_{1} \nu_{2}<1$. By inspection $\sigma>30$ so that at least one root of the quadratic for $\alpha$ exceeds 15 . But $0 \leqq \nu_{j} \leqq 1$ so

$$
|\alpha| \equiv \nu_{4} \nu_{5} \nu_{6}+\nu_{3} \nu_{5} \nu_{6}+\nu_{3} \nu_{4} \nu_{6}+\nu_{3} \nu_{4} \nu_{5} \leqq 4
$$

Hence $\alpha$ is determined by the quadratic. When $\alpha$ is known $\beta$ can be found from the linear equation. So all the coefficients of the quartic whose roots are the $\nu_{j}(j=3, \cdots, 6)$ are determined. In other words, the hypotheses of the Remark ensure that the $\nu_{j}^{\prime}(j=1, \cdots, 6)$ are the $v_{j}^{\prime \prime}(j=1, \cdots, 6)$ in some order.

This result shows that a formal solution obtained by the methods of Section 6.3 will correspond to a real set of points on the sphere if and only if the roots of the sextic are real and lie in $[0,1]$ and two of the roots are icosahedral co-ordinates of some point. This is what actually happened in the case $\left(h=h^{\prime}=1\right)$ which we investigated.

6.42. Transformation to Cartesian co-ordinates. The transformation from icosahedral co-ordinates to Cartesian co-ordinates with the center of the sphere as origin is achieved as follows. The 15 diameters of the sphere through mid-points of opposite edges of the icosahedron form 5 sets of 3 mutually perpendicular axes. Choose one set $(O A, O B, O C)$ as axes of co-ordinates (Figure 2 ).

Since $A, B, C$ are $(1,0,0),(0,1,0)$ and $(0,0,1)$ respectively then the icosahedral

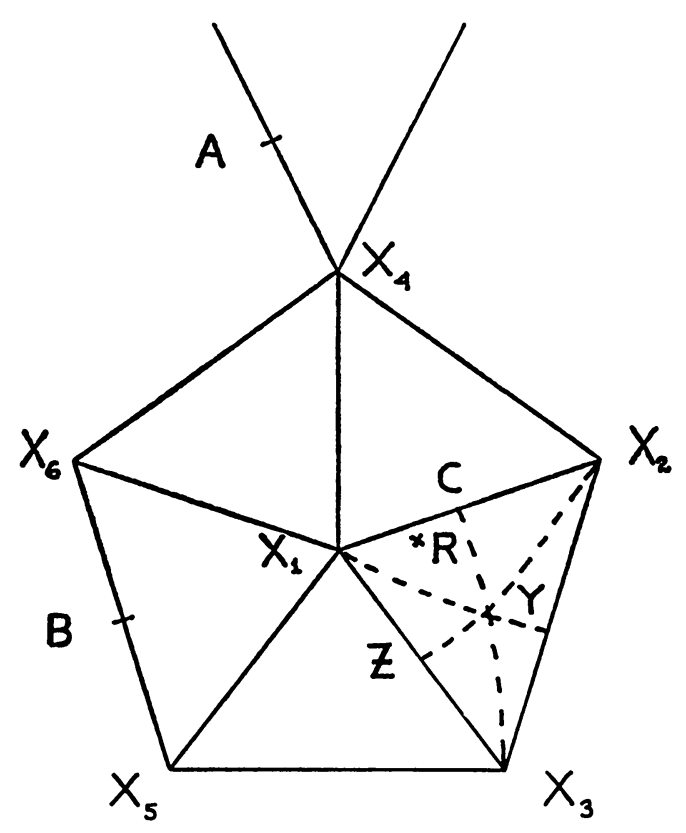

FIG. 2 
vertices $X_{1}, X_{2}, \cdots, X_{6}$ are respectively

$(0, \lambda, \rho) ; \quad(0,-\lambda, \rho) ; \quad(-\rho, 0, \lambda) ; \quad(\rho, 0, \lambda) ; \quad(-\lambda, \rho, 0) ; \quad(\lambda, \rho, 0)$

where

$$
\lambda \equiv \sqrt{\frac{\tilde{j}-\sqrt{ } 5}{10}} \text { and } \rho \equiv \sqrt{\frac{\tilde{5}+\sqrt{ } \tilde{5}}{10}}
$$

were computed in Section 6.1.

Now consider an arbitrary point $R \equiv(\alpha, \beta, \gamma)$ in triangle $X_{1} Y C$. It is clear on inspection of Figure 2 that the vertices $X_{1}, X_{2}, \cdots, X_{6}$ are ranked in order of increasing distance from $R$. So if $\nu_{1}, \nu_{2}, \cdots, \nu_{6}$ are the icosahedral co-ordinates of $R$ in decreasing order of magnitude and $\mu_{j} \equiv \nu_{j}^{1 / 2}(j=1, \cdots, 6)$ then

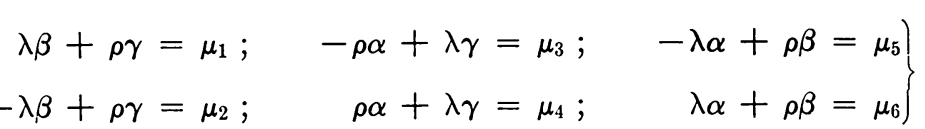

So the Cartesian co-ordinates $(\alpha, \beta, \gamma)$ of $R$ can be found in terms of the icosahedral co-ordinates.

We have almost immediately the following necessary and sufficient condition for arbitrary positive numbers $\nu_{j}^{\prime}(j=1, \cdots, 6)$ in decreasing order of magnitude to be the icosahedral co-ordinates of some point on the sphere.

$$
\left.\begin{array}{c}
\sum_{j=1}^{6} \nu_{j}^{\prime}=2 \\
\frac{\mu_{1}^{\prime}+\mu_{2}^{\prime}}{\mu_{3}{ }^{\prime}+\mu_{4}{ }^{\prime}}=\frac{\mu_{3}^{\prime}-\mu_{4}^{\prime}}{\mu_{5}{ }^{\prime}-\mu_{6}^{\prime}}=\frac{\mu_{5}^{\prime}+\mu_{6}^{\prime}}{\mu_{1}^{\prime}-\mu_{2}^{\prime}}=\frac{\rho}{\lambda} \equiv \frac{1}{2}(1+\sqrt{ } 5)
\end{array}\right\}
$$

where as usual $\mu_{j}^{\prime} \equiv\left(\nu_{j}^{\prime}\right)^{1 / 2}$ for $j=1, \cdots, 6$.

This is an alternative criterion to the Remark of Section 6.41. It differs from the Remark in that in practice it cannot be applied exactly, for the roots of a sextic equation can normally be found only approximately. So a very slight deviation of any of the three quotients from $\frac{1}{2}(1+\sqrt{ } 5)$ would pass undetected.

The criterion is useful mainly as a numerical check on the calculations. When it is applied to the roots $\nu_{j}^{\prime}$ of the sextic equation of Section 5.3, $\sum \nu_{j}^{\prime}=2$ by construction and the three quotients are

$$
1.61803, \quad 1.6180, \quad 1.6180
$$

where the last digit in each case is doubtful. In fact $\frac{1}{2}(1+\sqrt{ } 5)=1.618035 \cdots$.

We saw above how to find the Cartesian co-ordinates of a point $R$ with given icosahedral co-ordinates. All the points of $\omega(R)$, and of the antipodal set, have the same icosahedral co-ordinates. If in Figure 2 we had taken $R$ to be in triangle $X_{1} Y Z$ instead of in triangle $X_{1} Y C$ we would have obtained the antipodal set which would be equivalent.

We show finally how to shorten the calculation of the Cartesian co-ordinates of all the points of $\omega(R)$. The four images of $R$ obtained by rotation about the axis through $X_{1}$ are found by permuting suitably the $\mu_{j}(j=2, \cdots, 6)$ of $(4)$. No more calculation is needed, by the following classical property of the regular solids which is described by Ledermann [6]. 
Five cubes are inscribed in the configuration of the icosahedron-dodecahedron. The rotations of the icosahedral group $a_{5}$ induce just the even permutations of these cubes. The number of rotations of $Q_{5}$ that keep a particular cube fixed is thus the number of even permutations of four objects, namely 12. So 12 of the 24 symmetry rotations of this cube are also symmetry rotations of the icosahedron. In fact they are the 12 symmetry rotations of the tetrahedron formed by any four nonadjacent vertices of the cube.

Now consider a general invariant set $\omega(R)$ of the icosahedral rotations. The 60 points of $\omega(R)$ must comprise just five general invariant sets of the tetrahedral rotations. So from any five points of $\omega(R)$ that are mutually inaccessible by these tetrahedral rotations it is possible to generate all the points of $\omega(R)$, simply by applying the tetrahedral substitutions.

The choice of $(O A, O B, O C)$ as axes of co-ordinates (Figure 2) amounts to choosing one of the five cubes. The three directions are perpendicular to its faces. None of the rotations of the icosahedron about the axis through $X_{1}$ leave this cube invariant; so $R$ and its four images whose Cartesian co-ordinates have been found by solving equations like (4) are mutually inaccessible by the tetrahedral rotations of this cube. The tetrahedral substitutions appropriate to rectangular axes $(O A$, $O B, O C$ ) are generated by

$$
(\alpha, \beta, \gamma) \rightarrow(\beta, \gamma, \alpha)
$$

and

$$
(\alpha, \beta, \gamma) \rightarrow(\alpha,-\beta,-\gamma)
$$

When $R$ and its four images have been identified it is thus simple to write down the Cartesian co-ordinates of all 60 points of $\omega(R)$.

The octahedral substitutions that generate $\omega(R)$ from $R$ when the formula is based on the octahedral group are the $3 \times 3$ pseudo-permutation matrices with determinant +1 .

The tetrahedral substitutions that generate $\omega(R)$ from five suitable representatives when the formula is based on the icosahedral group are those $3 \times 3$ pseudopermutation matrices with determinant +1 in which the number of negative elements is either two or none.

live suitable representatives of $\omega(R)$ for the 14th degree formula are

$$
\begin{array}{rrr}
(-0.15111, & 0.15524, & 0.97626) \\
(0.31584, & 0.25705, & 0.91334) \\
(0.34631, & 0.66628, & 0.66042) \\
(-0.10181, & 0.81739, & 0.56702) \\
(-0.40923, & 0.50155, & 0.76223) .
\end{array}
$$

7. Review. Some of the formulas have been obtained before by three other writers working independently. They are identified by initial in the last column of Table 2. Finden [2] considers, besides the regular solids, axially symmetric formulas of Cartesian-product type and stereographic images of Simpson's Rule in the plane. 
Sobolev [13] employs group characters to investigate some invariant networks of points which reduce in special cases to certain of our formulas. D. G. Kendall [5] has shown how to deduce others from formulas for the solid sphere of the type given by Ditkin [1] and by Hammer and Stroud [3]. Kendall's arguments work in both directions so that formulas for the surface may be combined to obtain formulas for the solid sphere-now the more natural sequence of reasoning.

The formulas listed in Table 2 of the present paper have been constructed to obtain maximum efficiency subject to invariance under the appropriate group of rotations. This invariance seems to be at worst a harmless requirement so that for practical purposes the problem of spherical integration is solved provided the number $N$ of sample points is within the range of Table 2 . The existence of larger formulas, of arbitrarily high degree $p$, that are efficient ( $E$ near unity) has not been proved although D. G. Kendall [5] has obtained $E=\frac{2}{3}$ for any odd value of $p$. This is achieved by means of axially symmetric Cartesian-product formulas which are derived from work of Peirce [9] on the spherical shell. The same idea was applied earlier by Ditkin [1] to the complete solid sphere.

In conclusion two alternative methods of obtaining indefinitely large formulas are suggested.

7.1. The Extremal Property. With the notation of Section 5, let $\sum a_{r}=\int d S=$ $4 \pi$. The conditions that an integration formula, not necessarily invariant, must satisfy for $p$ th degree accuracy are

$$
\sum_{r=1}^{t} \sum_{s=1}^{t} a_{r} a_{s} P_{m}\left(\cos \gamma_{r s}\right)=0 ; \quad m=1, \cdots, p .
$$

Because these expressions are non-negative definite this is equivalent to

$$
\sum_{r=1}^{t} \sum_{s=1}^{t} a_{r} a_{s} \sum_{m=0}^{p} \lambda_{m} P_{m}\left(\cos \gamma_{r s}\right)=16 \pi^{2} \lambda_{0}
$$

where the $\lambda_{m}(m=1, \cdots, p)$ are any positive constants. If

$$
L_{p}(\mu) \equiv \sum_{m=0}^{p} \lambda_{m} P_{m}(\mu)
$$

then to find a $p$ th degree formula we have simply to arrange that

$$
\sum_{r=1}^{t} \sum_{s=1}^{t} a_{r} a_{s} L_{p}\left(\cos \gamma_{r s}\right)
$$

attains its minimum $16 \pi^{2} \lambda_{0}$ with respect both to points $A_{r}$ and to weights $a_{r}$. The number of points must be large enough for the required integration formula to exist. $\frac{1}{3}(p+1)^{2}$ points may be sufficient while (almost any) $(p+1)^{2}$ points are certainly sufficient.

The polynomial $L_{p}(\mu)$ is highly arbitrary and it may be convenient to use the fact that the coefficients in the expansion of $\mu^{p}$ in Legendre polynomials $P_{m}(\mu)$ are alternately positive and zero. This follows from the recurrence relation

$$
(2 m+1) \mu P_{m}(\mu)=(m+1) P_{m+1}(\mu)+m P_{m-1}(\mu)
$$

and induction on $p$. 
If the points $A_{r}$ are accepted only in antipodal pairs, to eliminate the odd harmonics, then the condition for a $p$ th degree integration formula (where $p$ is even) is

$$
\sum_{r=1}^{t} \sum_{s=1}^{t} a_{r} a_{s}\left(\cos \gamma_{r s}\right)^{p}=\frac{16 \pi^{2}}{p+1}
$$

with $\sum a_{r}=4 \pi$.

Instead we may modify the criterion of efficiency, which emphasizes the lower harmonics to the extent of ignoring altogether those whose degree exceeds $p$. This can be done by choosing a convenient function $L_{\infty}(\mu)$ with a convergent expansion in Legendre polynomials that has positive coefficients, and minimizing

$$
\sum_{r=1}^{t} \sum_{s=1}^{t} a_{r} a_{s} L_{\infty}\left(\cos \gamma_{r s}\right)
$$

In a sequel to this paper the problem of spherical integration is restated in probabilistic terms and shown to lead to an extremal problem of just this form.

7.2. The Reproducing Icosahedron. In the main part of the paper (Sections 2-6) only integration formulas based on the regular solids were considered and they were judged by the efficiency $E$. In the preceding Section the notion of efficiency was modified to provide a less artificial criterion and the regular solids played no part at all. In this final Section the idea of efficiency is discarded altogether and appeal is made only to symmetry.

The theory of Section 2, on which we have relied so heavily has two serious limitations. One is its inability to treat more than one regular solid (with its dual) at a time, for it is fundamental that one fixed realization of the group $G$ is considered. Finden [2] considers three dodecahedra in a certain mutual orientation but this formula seems to be beyond the scope of Section 2 . The second limitation is the lack of further finite subgroups of the rotation group which might provide other regular solids. This apparent shortcoming is a valuable safeguard when icosahedra are reproduced over the sphere in the following manner.

We saw in Section 6.42 that just five cubes could be inscribed in the icosahedral configuration and that they were permuted evenly by its symmetry rotations. The vertices of the cubes coincide in pairs at the centroids of the faces of the icosahedron. The converse property associates with any cube just two icosahedra. They are permuted by the rotations of the cube.

We say two icosahedra are first neighbors if there is a cube inscribed in them both in the way just described. Clearly every icosahedron has exactly five first neighbors. We say (inductively) that two icosahedra are $j$ th neighbors $(j=2,3, \cdots)$ if there is an icosahedron that is a first neighbor of one and a $(j-1)$ th neighbor of the other. Two icosahedra are simply neighbors if for some $j=0,1,2, \cdots$ they are $j$ th neighbors. Thus "neighborhood" is an equivalence relation.

Let $I_{0}$ denote the twelve vertices of a certain icosahedron. Let $I_{j}(j=1,2, \cdots)$ denote the set of vertices of all the $j$ th neighbors of $I_{0}$. These sets $I_{j}$ may not be disjoint. We propose equally-weighted integration formulas based on sets of points 
of the following form

$$
K_{M} \equiv \bigcup_{j=0}^{M} I_{j}
$$

Consider the set of points on the sphere

$$
K_{\infty} \equiv \bigcup_{j=0}^{\infty} I_{j}
$$

It consists of the vertices of all icosahedra that are neighbors of $I_{0}$. Let $I_{0}^{\prime}$ be any such icosahedron. Because neighborhood is an equivalence relation $K_{\infty}$ is equally well described as consisting of the vertices of all neighbors of $I_{0}{ }^{\prime}$. So the group $G_{\infty}$ of rotations of the sphere under which the set $K_{\infty}$ is invariant includes those rotations that transform $I_{0}$ into any one of its neighbors. The ordinary symmetry rotations of $I_{0}$ are properly included so $G_{\infty}$ is too large to be one of the three finite groups associated with regular solids. $G_{\infty}$ is obviously not cyclic nor dihedral. Hence $G_{\infty}$ is not finite, by Weyl [15]. Hence $K_{\infty}$ is not a finite set. It is, of course, countable.

By suitable choice of $M$ the integration formula based on the set $K_{M}$ has an arbitrarily large number of points. The justification for using the ascending sequence of sets $K_{M}$ is simply the striking symmetry of their union $K_{\infty}$, as expressed by invariance under the group $G_{\infty}$. A high degree of symmetry in the integration formula is a reasonable aim because the integral itself is an invariant of the whole rotation group. The question of whether the points of $K_{M}$ are asymptotically distributed uniformly over the sphere, and associated matters of convergence are postponed to another occasion.

These formulas have two practical advantages. The sets $K_{M}$ are nested so that no work is wasted when a crude approximation to the integral is later refined. This applies if the sample points are found explicitly. In fact the second property removes the need for that if a computer is available. All that is necessary is a routine to locate the vertices of the five first neighbors of a given icosahedron. Sequential generation of the sample points should then be possible, followed at once by evaluation there of the integrand.

8. Acknowledgment. I am grateful to Professor D. G. Kendall for much stimulating discussion and for permission to mention unpublished work of his. I am obliged to the Department of Scientific and Industrial Research for a Research Studentship.

Statistical Laboratory,

Cambridge, England.

1. B. A. Ditkin, “Some approximate formulae for evaluating triple integrals," Dokl. Akad. Nauk SSSR, v. 62, 1948, p. 445-7.

2. C. Finden, Spherical integration, Dissertation submitted for the Diploma in Numerical Analysis and Automatic Computing, University of Cambridge, 1961.

3. P. C. Hammer \& A. H. Stroud, "Numerical evaluation of multiple integrals, II," $M T A C$, v. 12,1958 , p. $272-80$.

4. V. Heine, Group Theory in Quantum Mechanics, Pergamon, 1960.

5. D. G. Kendall, "Gaussian integration on the sphere," Unpublished Report to the Atlas Computer Laboratory, 1962.

6. W. LedermanN, Introduction to the Theory of Finite Groups, Oliver \& Boyd, 1949.

7. L. A. Lyusternik \& B. A. Ditkin, "Construction of approximate formulae for evaluat ing multiple integrals," Dokl. A kad. Nauk SSSR, v. 61, 1948, p. 441-4. 
8. L. A. Lyusternik, "Some cubature formulae for repeated integrals," Dokl. Akad. Nauk SSSR, v. 62, 1948, p. 449-52.

9. W. H. Peirce, "Numerical integration over the spherical shell," MTAC, v. 11, 1957, p. 244-9.

10. S. L. Sobolev, "Formulas for mechanical cubature in $n$-dimensional space," Dokl. Akad. Nauk SSSR, v. 137, 1961, p. 527-30.

11. S. L. SobolEv, "Different convergence types of cubature and quadrature formulas," Dokl. Akad. Nauk SSSR, v. 146, 1962, p. 41-2.

12. S. L. Sobolev, "Cubature formulas on a sphere invariant with respect to any finite group of rotations," Dokl. Akad. Nauk SSSR, v. 146, 1962, p. 310-3.

13. S. L. Sobolev, "On the number of nodes of cubature formulae on a sphere," Dokl. Akad. Nauk SSSR, v. 146, 1962, p. 770-3.

14. G. Szegö, Orthogonal Polynomials, Colloquium Publications, v. 23, American Mathematical Society, 1959.

15. H. Weyl, Symmetry, Princeton University Press, N. J., 1952, p. 149-54.

Note. Four papers, those of Lyusternik and Ditkin [7], Lyusternik [8], and Sobolev [10, 11] are not mentioned in the text, but appear in this list of references because they are concerned with the approximation of multiple integrals. 